\title{
Generation of relativistic vortex laser beams by spiral shaped plasma
}

\author{
Tianyun Long $\odot,{ }^{1,2}$ Cangtao Zhou, ${ }^{2, *}$ Libao Ju, ${ }^{2}$ Taiwu Huang, ${ }^{2}$ Mingyang $\mathrm{Yu},{ }^{2}$ Ke Jiang, ${ }^{2,3} \mathrm{Chaoneng} \mathrm{Wu},{ }^{2,3}$ Sizhong Wu, ${ }^{2}$ \\ Hua Zhang, ${ }^{2}$ Bin Qiao, ${ }^{1,2}$ Shuangchen Ruan ${ }^{\circ},{ }^{2}$ and Xiantu $\mathrm{He}^{1,2,4, \dagger}$ \\ ${ }^{1}$ Center for Applied Physics and Technology, HEDPS, and School of Physics, Peking University, Beijing 100871, China \\ ${ }^{2}$ Center for Advanced Material Diagnostic Technology, and College of Engineering Physics, \\ Shenzhen Technology University, Shenzhen 518118, China \\ ${ }^{3}$ Graduate School, China Academy of Engineering Physics, Beijing 100088, China \\ ${ }^{4}$ Collaborative Innovation Center of IFSA (CICIFSA), Shanghai Jiao Tong University, Shanghai 200240, China
}

(Received 10 November 2019; accepted 8 July 2020; published 27 July 2020)

\begin{abstract}
Three-dimensional particle-in-cell simulations show that relativistic Gaussian laser light can be transformed into relativistic vortex laser light with axial orbital angular momentum (OAM) as it propagates through a homogeneous spiral-profiled low-density plasma slab. In the process, the plane equiphase surfaces of the Gaussian laser undergo azimuthal modulation and become a continuous helical surface. The intensity profile of the laser changes from maximum-on-axis to donut-shaped. Because of the azimuthally varying slab thickness, the laser ponderomotive and the charge-separation forces exert a torque on the plasma when it passes through it, resulting in the creation of oppositely directed OAM in the plasma ions and the laser light, with the electrons remaining nearly OAM free. The proposed scheme can be used to generate both single- and multi-mode relativistic vortex lasers propagating along the direction of the input laser, which is especially convenient for many applications.
\end{abstract}

DOI: 10.1103/PhysRevResearch.2.033145

\section{INTRODUCTION}

Depending on the light polarization and azimuthal phase profile, the total angular momentum (AM) of light consists of spin angular momentum (SAM) and orbital angular momentum (OAM) [1]. It is well known that circularly polarized (CP) light carries SAM, with $\pm \hbar$ per photon for right- and left-handed CP light, respectively [2,3]. More recently, Allen et al. [4] showed that nonplanar laser light with nonaxisymmetric field gradients possesses OAM. In particular, an ideal Laguerre-Gaussian pulse with helical phase dependence $\exp (-i l \varphi-i k x)$ has a well-defined average OAM value $l \hbar$ per photon, where $k$ is the wave vector in the axial or propagation direction $x, \phi$ is the azimuthal angle, and $l$ is a mode index, or the topological charge.

The OAM of light has drawn much attention because of its novel characteristics [5]. In particular, the light OAM introduces a new degree of freedom in laser-particle interaction, leading to applications such as optical spanners [6], angular Doppler shift [7], light AM entanglement [8], and spectroscopic probe of magnetic excitations [9]. With the rapid development of intense-laser technology, relativistic vortex lasers have further broadened the applications, such as for positron and electron acceleration [10], OAM transfer to matter [11,12], AM oscillations [13], realization of

\footnotetext{
*zcangtao@sztu.edu.cn

†xthe@iapcm.ac.cn
}

Published by the American Physical Society under the terms of the Creative Commons Attribution 4.0 International license. Further distribution of this work must maintain attribution to the author(s) and the published article's title, journal citation, and DOI. relativistic electron vortices $[14,15]$, generation of intense high-order vortex harmonics [16,17], OAM transfer to gamma-ray beams [18,19], realization of enduring strong magnetic fields [20], etc. Accordingly, generation of intense vortex laser light has become an important topic of research, and several methods have been proposed, including the use of computer generated holograms [21], cylindricallens mode convertors [22], spiral phase plates [23], and $q$ plates [24]. However, for relativistic-intense laser light, most such methods are limited by the ionization threshold of the material used, such that destructive optical breakdown can occur. Recently, several novel schemes have been proposed, such as the use of light fan [25], plasma hologram [26], and stimulated Raman scattering [27]. The first two produce relativistic vortex beams in the reflection direction, and the last one in the transmission direction but involves multiple laser beams. These properties can lead to undesirable effects, such as interference by the incident laser(s).

Here we propose a scheme for obtaining relativistic vortex light in the same direction of the incident laser. The latter, of high intensity and arbitrary polarization, irradiates a low-density plasma slab with azimuthally varying thickness, which modulates and warps the initially plane light-wave phases into a continuous spiral structure. Depending on the spiral structure of the plasma slab, both single- and multimode vortex light can be generated.

\section{SINGLE VORTEX MODE GENERATION}

The field amplitude of a vortex laser pulse, say with Laguerre-Gaussian distribution, propagating along the 


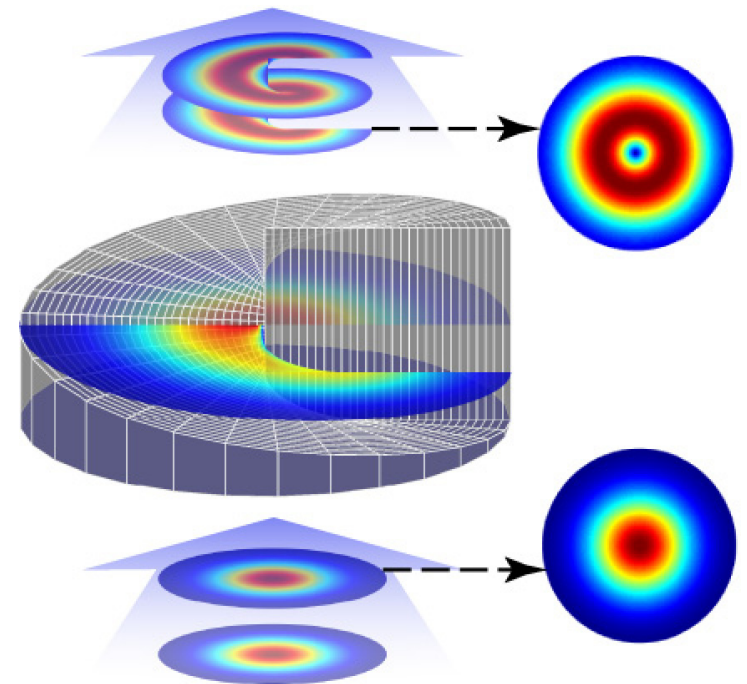

FIG. 1. The spiral phase plasma (center) slab that converts an $l=0$ (vortex-free Gaussian) laser into an $l=1$ vortex laser. Representative equiphase surfaces of the input and output light are shown in color, with the deepest red and deepest blue colors indicating the maximum and null intensity values, respectively. Projections of the equiphase surfaces in the transverse plane are shown on the right.

$x$ direction is given in cylindrical coordinates $(r, \phi, x)$ by

$$
\begin{aligned}
L G_{p l}(r, \phi, x)= & c_{p l} \frac{w_{0}}{w}\left(\frac{r \sqrt{2}}{w}\right) \exp \left(\frac{-r^{2}}{w^{2}}\right) L_{p}^{|l|}\left(\frac{2 r^{2}}{w^{2}}\right) \\
& \times \exp (-i l \phi) \exp \left(\frac{-i k r^{2}}{2 R}\right) \exp (i \psi)
\end{aligned}
$$

where $L_{p}^{l}(x)$ is the generalized Laguerre polynomial with $p$ and $l$ the radial and azimuthal mode indices, $c_{p l}$ the normalization coefficient, $r$ the radius, $w_{0}$ the beam waist, $w=$ $w_{0} \sqrt{1+\left(x / x_{R}\right)^{2}}$ the beam radius, $x_{R}=\pi w_{0}^{2} / \lambda$ the Rayleigh range, $\lambda$ the wavelength, $k$ the wave number, $R=\left(x^{2}+x_{R}^{2}\right) / x$ the radius of curvature of the phase fronts, $\psi=(2 p+|l|+$ $1) \arctan \left(x / x_{R}\right)$ the Gouy phase. Here, $l$ is the number of helices of the helical wavefront, or OAM of $l \hbar$ per photon, $p$ is the radial mode number (corresponding to $p+1$ concentric rings with zero on-axis if $l>0$ ). The $L G_{00}$ mode is the usual Gaussian.

The proposed scheme for transforming an OAM-free (corresponsing to the $L G_{00}$ mode, or light with Gaussian transverse profile) laser pulse into a vortex laser is schematically illustrated in Fig. 1. The thickness of the uniform-density plasma slab is given by $h(\phi)=h_{0}(2 \pi-\phi) / 2 \pi$, where $h$ decreases in the anticlockwise direction from the maximum value $h_{0}$ to zero, having a jump at $\phi=0$ (or $\left.2 \pi\right)$. The incident laser has a Gaussian $\left(L G_{00}\right)$ transverse profile, with maximum on-axis intensity and plane phase fronts separated in the axial direction by the laser wavelength $\lambda=2 \pi / k$. Since the phase velocity $v_{p}$ of light depends on the plasma density and is larger than the vacuum light speed $c$, the laser pulse exiting the plasma slab will have a helical phase front given by, say $\sim \exp (-i l \phi-i k x)$, where the topological charge $l$ is determined by the thickness profile of the slab. For example, to produce a vortex laser with given $l$, one should have

$$
\left(h_{0} / c-h_{0} / v_{p}\right) c=l \lambda, \text { or }
$$

$$
h_{0}=\left(1+\frac{c}{v_{p}-c}\right) l \lambda .
$$

The generated vortex light will have a negative topological charge if the plasma slab has an anticlockwise increasing thickness given by $h(\phi)=h_{0} \phi / 2 \pi$.

The light intensity and momentum are also redistributed during the phase modulation. To follow the changes, it is convenient to introduce the Poynting vector. For a beam with vector potential $\vec{A}=\left(\delta \vec{e}_{y}-i \sqrt{1-\delta^{2}} \vec{e}_{z}\right) u \exp (-i f) \exp (i \omega t)$, where $\vec{e}_{y, z}$ are the unit vectors, $\delta$ is the polarization, $u$ the field amplitude, $f$ the spatial phase, and $\omega$ the laser frequency. The corresponding period-averaged momentum density can be written as $\vec{P}=\vec{S} / c^{2}=\langle\vec{E} \times \vec{H}\rangle_{t} / c^{2}$, where $\vec{E}$ and $\vec{H}$ are the electric and magnetic fields and the energy flow density $\vec{S}$ is given by

$$
\vec{S}=\frac{1}{2 \mu_{0}}\left[u^{2} \omega \nabla f+\frac{1}{2} \sigma \omega\left(\partial_{y} u^{2} \vec{e}_{z}-\partial_{z} u^{2} \vec{e}_{y}\right)\right],
$$

where $\sigma=2 \delta \sqrt{1-\delta^{2}}$. We see that $\vec{S}$ depends on the phase gradient $\nabla f$. In particular, for linearly polarized (LP) light $(\sigma=0)$, we have $\vec{S}=\frac{1}{2 \mu_{0}} u^{2} \omega \nabla f$, i.e., the direction of $\vec{S}$ is only determined by the phase gradient. For the $L G_{00}$ mode, there is no average transverse momentum, but for the vortex modes both the radial and azimuthal momenta exist. The corresponding transverse light intensity distribution is annular, with a phase singularity on the axis, as shown in Fig. 1.

To understand the interaction in more detail, in particular, how the OAM is generated and conserved, numerical simulations are carried out using the fully relativistic particlein-cell code $\mathrm{EPOCH}$ [28]. A $0.8-\mu \mathrm{m}, p$-polarized Gaussian laser with $4-\mu \mathrm{m}$ focal radius is incident along the $x$ axis from the left boundary at $t=0$. The laser peak intensity is $2.14 \times 10^{18} \mathrm{~W} / \mathrm{cm}^{2}$, corresponding to the laser parameter $a_{0}=e E_{L} / m_{e} c \omega=1$, where $E_{L}$ is the peak laser electric field, $e$ and $m_{e}$ the electron charge and mass. The temporal profile of the laser is given by $\exp \left[-\left(t-t_{0}\right)^{2} / \Delta t^{2}\right]$, centered at $t_{0}=10 T_{0}$. The pulse width is $\Delta t=5 T_{0}$, where $T_{0}$ is laser period. The spiral plasma slab contains protons and electrons, and is of uniform density $0.1 n_{c}$, where $n_{c}=\varepsilon_{0} m_{e} \omega^{2} / e^{2}=$ $1.7 \times 10^{21} \mathrm{~cm}^{-3}$ is the critical density and $\varepsilon_{0}$ the vacuum permittivity. The phase velocity of intense light propagating in an underdense plasma is $v_{p}=c / \sqrt{1-n_{e} / \gamma_{L} n_{c}}$ [29], where $n_{e}$ is the plasma density and $\gamma_{L}=\sqrt{1+a_{0}^{2} / \alpha}$ is the relativistic parameter with $\alpha=1$ for CP and $\alpha=2$ for LP light. Therefore, from Eq. (2) with $l=1$, the maximum thickness of the spiral plasma is $h_{0}=19 \mu \mathrm{m}$. The plasma slab, with radius $11 \mu \mathrm{m}$, is located in $0<x<h_{0}$ and has an anticlockwise decreasing thickness. The simulation box is in $-10 \mu \mathrm{m}<$ $x<35 \mu \mathrm{m}$ and $-12 \mu \mathrm{m}<(y, z)<12 \mu \mathrm{m}$. The simulation cell size is $0.05 \mu \mathrm{m}$ in all three Cartesian directions, and per cell there are 10 each of macro electrons and ions.

The electric field $E_{y}$ along $\left(y, z_{1,2}\right)=(0, \mp 2) \mu \mathrm{m}$ in the $\left(\xi=x-c t+x_{0}, y, z\right)$ frame, where $x_{0}=18 \mu \mathrm{m}$, for the slab thicknesses 4.75 and $14.25 \mu \mathrm{m}$, respectively, is shown in Fig. 2(a). When the laser enters the plasma, the local $v_{p}$ becomes larger than $c$ but $v_{g}=c^{2} / v_{p}<c$. At $34 T_{0}$, the exiting laser light at $z_{1}$ has $v_{p}$ less than that at $z_{2}$, causing a phase 

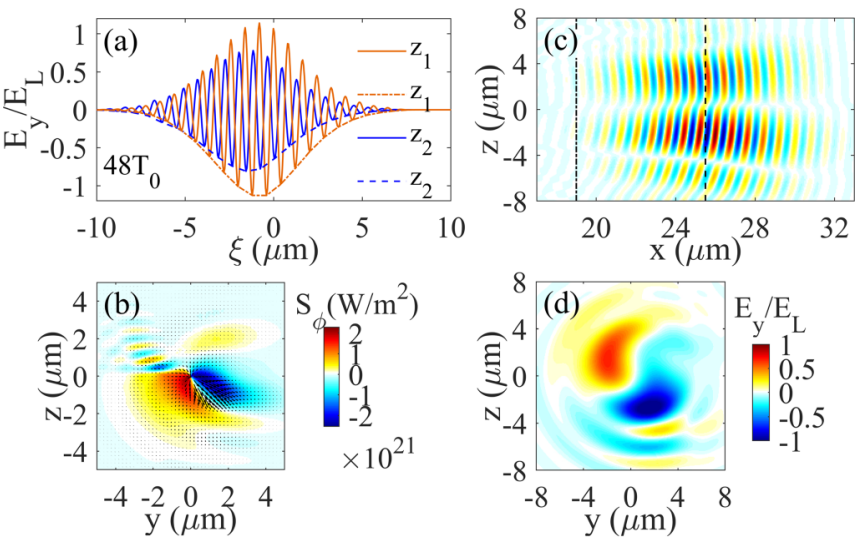

FIG. 2. (a) Normalized electric field comoponet $E_{y} / E_{L}$ along the lines $\left(x, 0, z_{1,2}\right)$, where $z_{1,2}=\mp 2 \mu \mathrm{m}$, at $48 T_{0}$ in the $(\xi, y, z)$ frame. (b) Poynting vector at $x=9.5 \mu \mathrm{m}$ and $35 T_{0}$, where the azimuthal $S_{\phi}$ is color coded and the radial component $S_{r}$ represented by black arrows. The maximums of these components are of the same order of magnitude. (c) $E_{y} / E_{L}$ in the $y=0$ plane at $56 T_{0}$, when the main part of the laser has just left the right boundary (marked by the dash-dot line) of the slab. (d) $E_{y} / E_{L}$ in the transverse plane at $x=25.5 \mu \mathrm{m}$, marked by a dashed line in (c).

difference. When both of them have exited the plasma at $48 T_{0}$, they will have a $\pi$ phase difference, and the originally separated equiphase surfaces become warped and join together to form a continuous helical surface. From Eq. (3) and Fig. 2(b), one can see that in the process the resulting gradient of the spatial phase $f$ leads to azimuthal as well as radial momentum flow, and the positive component plays a leading role. Due to the accompanying energy flow, there is also a slight amplitude difference at $z_{1}$ and $z_{2}$, as can be seen from the wave envelopes in Fig. 2(a). The output laser at $56 T_{0}$ is shown in Fig. 2(c). Figure 2(d) suggests that at $x=25.5 \mu \mathrm{m}, E_{y}$ has a helical structure. The output vortex laser light can be expressed in terms of the LG modes as $0.12 L G_{00}+0.79 e^{i \phi_{1}} L G_{01}+0.13 e^{i \phi_{2}} L G_{02}$, with $\phi_{1} \sim 1.2$ and $\phi_{2} \sim 4$.2. Clearly, the $L G_{01}$ component dominates, in agreement with the theory above.

Simulations also show that with the OAM generation in the laser during the laser-plasma interaction, the plasma protons acquire nearly equal amount of OAM in the opposite direction, with the electrons remaining nearly OAM free. On the other hand, the protons gain less than $0.1 \%$, and the electrons around $4 \%$, of the laser energy. In the interaction, the maximum proton velocity is around $0.001 \mathrm{c}$ and their displacement is much less than a wavelength. Figure 3 shows the evolution of the momentum of the plasma particles and the laser light. In the $x, y$ plane at $z=-2 \mu \mathrm{m}$. At first, both the electrons and protons gain (oscillating) momentum along the polarization direction $y$, which can be attributed to the intense laser electric field. After the laser has passed through the spiral shaped plasma slab, the proton momentum distribution in this direction becomes asymmetric: positive in the region $y>0$ and negative in $y<0$. However, $P y_{e}$ remains oscillating. Thus, after summing over the slab the ions have negative net $y$-momentum gain and electrons remain $y$-momentum free, as can be seen in Figs. 3(a)-3(c). The
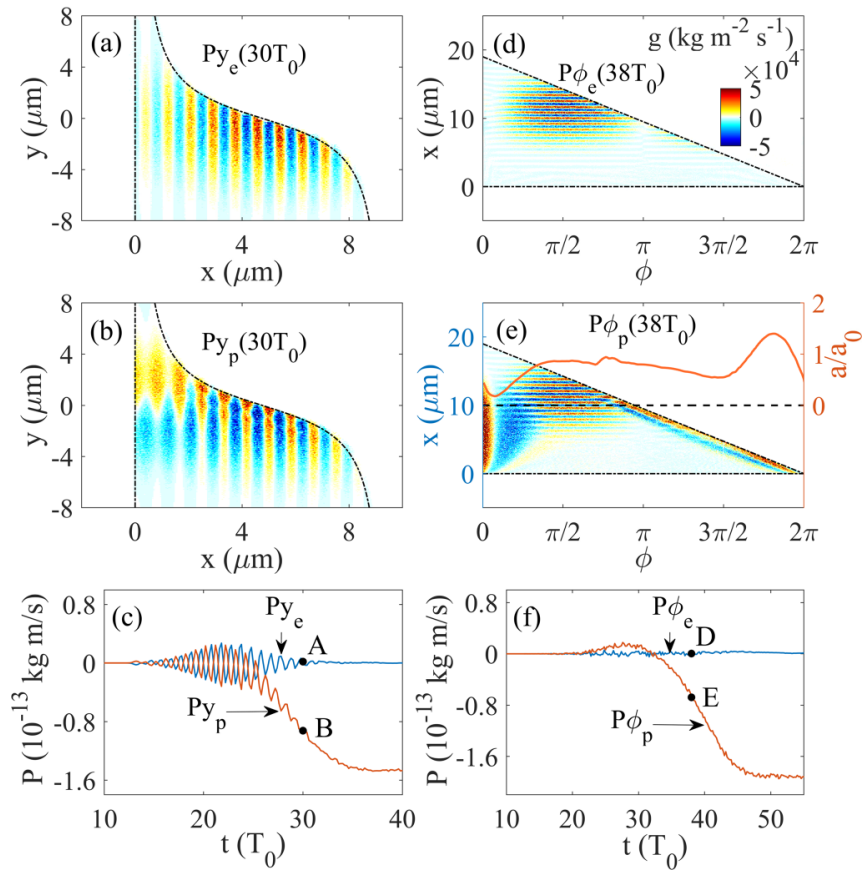

FIG. 3. (a) and (b) Instantaneous proton and electron $y$ component momentum density $P_{y_{e, p}}$ on the plane $z=-2 \mu \mathrm{m}$ at $30 T_{0}$. (c) Evolution of the total $P_{y_{e, p}}$ in a slice of one wavelength thickness centered at $z=-2 \mu \mathrm{m}$, where the points $\mathrm{A}$ and $\mathrm{B}$ correspond to (a) and (b), respectively. (d) and (e) Instantaneous proton and electron azimuthal momentum density $P_{\phi_{e, p}}$ at $r=2 \mu \mathrm{m}$ at $38 T_{0}$. The orange curve in (e) shows the normalized wave electric field amplitude along $x=10 \mu \mathrm{m}$ denoted by the black dashed line. (f) Evolution of total $P_{\phi_{e, p}}$ in the slice of one wavelength thickness centered at $r=2 \mu \mathrm{m}$, where points D and E correspond to (d) and (e), respectively. (a), (b), (d) and (e) share the same color bar, shown in (d), and the black dash-dot lines outline the plasma boundary.

peculiar momentum gain by the plasma particles can be attributed to the displacement of electrons by the relativistic ponderomotive force $\sim-m_{e} c^{2} \nabla\left(1+e^{2}|\vec{A}|^{2} / \alpha m_{e}^{2} c^{2}\right)^{1 / 2}$ [30]. The ponderomotive force on the protons is negligible. However, the electron displacement gives rise to a strong charge separation field that strongly drives the protons, which gain $y$ momentum.

The OAM creation mechanism is similar to that of the azimuthal momentum, and is represented in Figs. 3(d)-3(f) for the azimuthal momentum density distribution in the cylindrical $\phi, x$ surface at $r=2 \mu \mathrm{m}$. The passage of the laser through the plasma slab results in appearance of a phase gradient and redistribution of the radial and azimuthal momentum and intensity of the laser light. The orange curve in Fig. 3(e) shows the field strength at $x=10 \mu \mathrm{m}$ and its gradient in $0<\phi<\pi / 2$ is mainly positive, corresponding to a negative azimuthal ponderomotive force on the plasma. As discussed, the latter leads to a negative azimuthal proton momentum density $P_{\phi_{p}}$ through the charge separation force. As the laser propagates through the plasma, the net azimuthal momentum of protons accumulates to a large value, but the momentum gain buy the electrons is negligible, as Figs. 3(d) and 3(f) show. $P_{\phi_{p}}$ contributes positively to the total proton OAM, and equal but opposite OAM is gained by the laser light. 

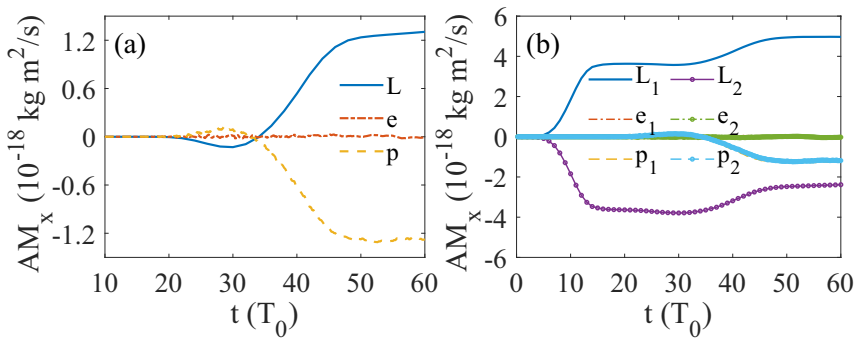

FIG. 4. (a) Evolution of total axial AM for the LP laser field, electrons and protons, denoted by L, e, and p, respectively. (b) Same as (a), but for right- and left-handed CP (denoted by the subscripts 1 and 2, respectively) lasers incident on the same spiral plasma slab. The curves for $e_{1}$ and $e_{2}$ nearly overlap, same for $p_{1}$ and $p_{2}$.

For completeness, we have also considered the OAM generation process for circularly polarized lasers. The axial AM of the pure $L G_{p l}$ mode with laser energy $\mathcal{E}$ is $\mathcal{A}_{x L}=\mathcal{E}(l+$ $\sigma) / \omega$, where $\sigma=0,1,-1$ for LP, right- and left-handed CP lasers. The AM of electrons, protons and fields are calculated by integrating AM flux $\vec{r} \times \vec{p}_{e, i}$ and $\epsilon_{0} \vec{r} \times(\vec{E} \times \vec{B})$ over the entire simulation box, and their evolution is presented in Fig. 4(a). We see that there is a small negative deviation for laser $\mathcal{A}_{x}$ and positive deviation for proton $\mathcal{A}_{x}$, as well as the small positive proton momentum deviation in Fig. 3(f), when laser begins to exit from the plasma. The reason is that when the laser equiphase surfaces are modulated, the negative azimuthal field momentum flux dominates over the positive component. With further modulation, the positive momentum flux begins to dominate, as can be seen in Fig. 2(d), and the total $\mathcal{A}_{x}$ of the laser fields increases gradually and reach a large value of about $1.3 \times 10^{-18} \mathrm{~kg} \mathrm{~m}^{-2} \mathrm{~s}^{-1}$. Additional OAM from the spiral plasma can also be added to the original SAM for both the left- and right-handed CP incident lasers, as shown in Fig. 4(b).

\section{MULTIPLE VORTEX MODE GENERATION}

With appropriate profile, say determined from the equiphase surface analysis, a spiral plasma can produce both single and multiple vortex modes. When the spiral plasma has a shape of $h(\phi)=h_{0}(2 \pi-\phi) / 2 \pi$ with $h_{0}=38 \mu \mathrm{m}$, simulations indicate that $L G_{02}$-like mode is obtained.

Recently, it is found that nonpure multiple vortex modes, such as superposition of two beams with well-defined OAM, convey an unexpectedly rich scenario in high-order harmonic generation [31], where use of nonpure vortex drivers increases dramatically the OAM content of the harmonics. For a mixed mode of $A_{1} \exp [i(\omega t-k x)] L G_{01}+A_{2} \exp$ $(i \Delta \varphi) \exp [i(\omega t-k x)] L G_{02} \sim\left[A_{1} \exp (-i \phi)+A_{2}(r / w) \exp \right.$ $(i \Delta \varphi) \exp (-i 2 \phi)] \exp [i(\omega t-k x)]=A \exp [-i f(r, \phi)] \exp [i$ $(\omega t-k x)$ ], where $A_{1,2}$ and $A$ denote amplitude, $\Delta \varphi$ the initial phase difference between these two modes, and $f(r, \phi)$ the phase satisfies $\tan [f(r, \phi)]=-\left[A_{1} \sin (-\phi)+\right.$ $\left.A_{2}(r / w) \sin (\Delta \varphi-2 \phi)\right] /\left[A_{1} \cos (-\phi)+A_{2}(r / w) \cos (\Delta \varphi-\right.$ $2 \phi)$ ], where $f(r, \phi)=l \phi$ for pure mode. The spiral plasma should have the shape $h(r, \phi)=x(r, \phi)$ from solving $f(r, \phi)+k x \equiv C$, where $\mathrm{C}$ is arbitrary phase constant. For simplicity, we make the minimum plasma thickness $h(r, \phi)_{\min }=0$ and $f(r, \phi)$ continuous about $\phi$. Figure 5(a) (a)

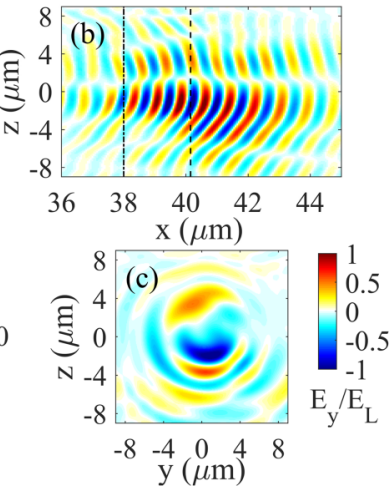

FIG. 5. (a) Spiral shaped plasma for superposed vortex mode generation. (b) $E_{y} / E_{L}$ map for $y=0$ plane when the laser is completely emergent. The black dot dashed line indicates the plasma right boundary. (c) $E_{y} / E_{L}$ map at transverse plane $(x=40.15 \mu \mathrm{m})$ denoted by the dashed line at (b).

shows the spiral shaped plasma for the superposition mode with $w=4 \mu \mathrm{m}, A_{2} / A_{1}=1$, and $\Delta \varphi=\pi / 4$. It is evident that for $r / w \ll 1$ and $r / w \gg 1$, the plasma tends to present the shape for pure $L G_{01}$ and $L G_{02}$ mode generation accordingly, which is resonable for $L G_{02}$ mode has an additional $r / w$ dependence compared to $L G_{01}$ mode. The maximum thickness can be gained from Eq. (2) with $l=2$ that $h_{0}=38 \mu \mathrm{m}$, and the other parameters remain the same.

The output vortex laser electric field $E_{y}$ is shown in Figs. 5(b) and 5(c), which has a total axial OAM of about $1.2 \times 10^{-18} \mathrm{~kg} \mathrm{~m}^{-2} \mathrm{~s}^{-1}$. Decomposing the output vortex beam indeed presents a superposition of $0.29 L G_{00}+$ $0.38 e^{i \phi_{1}} L G_{01}+0.32 e^{i \phi_{2}} L G_{02}$, where $\phi_{1} \approx 2.4$ and $\phi_{2} \approx 3.7$, indicating the superposition mode is realized. Other desired nonpure multiple modes can be generated directly in the same way.

\section{DISCUSSION AND CONCLUSION}

In the proposed scheme, the density of the plasma is important. We found that when $n_{e} \leqslant 0.1 n_{c}$, laser energy dissipation in the plasma will remain less than $5 \%$. But for $n_{e}>0.2 n_{c}$ the dissipation loss can be more than several tens percent. In addition, electron cavitation [29] must be avoided. Balancing laser ponderomotive force $f_{p}$ with plasma charge separation force $e E_{s}$ with charge separation field $E_{s}=n_{e} e r / 2 \epsilon_{0}$, for an incident laser with Guassian transverse profile $a=$ $a_{0} \exp \left(-r^{2} / w^{2}\right)$, noncavitation requires $n_{e} / n_{c}>\eta$, where

$$
\eta=\frac{1}{\pi^{2}} \frac{\lambda^{2}}{w^{2}} \frac{a_{0}^{2} / \alpha}{\sqrt{1+a_{0}^{2} / \alpha}} .
$$

In the simulation for LP $L G_{01}$ mode generation here, $\eta=$ $0.0017 \ll n_{e} / n_{c}=0.1$. For fixed $n_{e}$ and $\eta$, large $a_{0}$ would require a large beam radius in order to maintain a moderate $f_{p}$. For example, for $n_{e}=0.1 n_{c}$, simulations show that vortex lasers of $L G_{01}$-like modes can be generated with $a_{0}=0.1$ (and $h_{0}=16 \mu \mathrm{m}, w_{0}=4 \mu \mathrm{m}$ ) and $a_{0}=5$ (and $h_{0}=58 \mu \mathrm{m}$, $\left.w_{0}=12 \mu \mathrm{m}\right)$.

In summary, an underdense plasma of spiral thickness is proposed for relativistic vortex laser light generation. The 
slab plasma can be fabricated from aerogel [32] using 3D printing [33]. Upon full ionization, the lightest aerogel is of density $0.028 n_{c}$. As the laser passes through the plasma, it and the plasma proton gain equal and oppositely directed axial OAM. The scheme is simple, robust, and flexible and can produce laser beams with single and multiple vortex modes. Moreover, the generated vortex beam is in the transmission direction, so that in specific applications additional target(s) can be attached behind the spiral slab. Due to the robustness and transmissive characteristics, the spiral shaped plasma scheme may well facilitate the vortex laser related experimental studies.

\section{ACKNOWLEDGMENTS}

This work is supported by the National Key R\&D Program of China (2016YFA0401100); National Natural Science Foundation of China (NSFC) (11575031, 11575298, 11705120); and National ICF Committee in China.
[1] S. M. Barnett, J. Opt. B 4, S7 (2001).

[2] J. H. Poynting, Proc. R. Soc. London A, Ser. A 82, 560 (1909).

[3] R. A. Beth, Phys. Rev. 50, 115 (1936).

[4] L. Allen, M. W. Beijersbergen, R. J. C. Spreeuw, and J. P. Woerdman, Phys. Rev. A 45, 8185 (1992).

[5] A. M. Yao and M. J. Padgett, Adv. Opt. Photon. 3, 161 (2011).

[6] N. B. Simpson, K. Dholakia, L. Allen, and M. J. Padgett, Opt. Lett. 22, 52 (1997).

[7] J. Courtial, D. A. Robertson, K. Dholakia, L. Allen, and M. J. Padgett, Phys. Rev. Lett. 81, 4828 (1998).

[8] A. Mair, A. Vaziri, G. Weihs, and A. Zeilinger, Nature (London) 412, 313 (2001).

[9] A. A. Sirenko, P. Marsik, C. Bernhard, T. N. Stanislavchuk, V. Kiryukhin, and S.-W. Cheong, Phys. Rev. Lett. 122, 237401 (2019).

[10] J. Vieira and J. T. Mendonça, Phys. Rev. Lett. 112, 215001 (2014).

[11] G. Pariente and F. Quéré, Opt. Lett. 40, 2037 (2015).

[12] W. Wang, B. Shen, X. Zhang, L. Zhang, Y. Shi, and Z. Xu, Sci. Rep. 5, 8274 (2015).

[13] W. Gong, B. Shen, L. Zhang, and X. Zhang, New J. Phys. 21, 043022 (2019).

[14] J. Vieira, J. T. Mendonça, and F. Quéré, Phys. Rev. Lett. 121, 054801 (2018).

[15] L. B. Ju, C. T. Zhou, K. Jiang, T. W. Huang, H. Zhang, T. X. Cai, J. M. Cao, B. Qiao, and S. C. Ruan, New J. Phys. 20, 063004 (2018).

[16] X. Zhang, B. Shen, Y. Shi, X. Wang, L. Zhang, W. Wang, J. Xu, L. Yi, and Z. Xu, Phys. Rev. Lett. 114, 173901 (2015).

[17] A. Denoeud, L. Chopineau, A. Leblanc, and F. Quéré, Phys. Rev. Lett. 118, 033902 (2017).

[18] Y.-Y. Chen, J.-X. Li, K. Z. Hatsagortsyan, and C. H. Keitel, Phys. Rev. Lett. 121, 074801 (2018).
[19] L. B. Ju, C. T. Zhou, T. W. Huang, K. Jiang, C. N. Wu, T. Y. Long, L. Li, H. Zhang, M. Y. Yu, S. C. Ruan et al., Phys. Rev. Appl. 12, 014054 (2019).

[20] Y. Shi, J. Vieira, R. M. G. M. Trines, R. Bingham, B. F. Shen, and R. J. Kingham, Phys. Rev. Lett. 121, 145002 (2018).

[21] N. R. Heckenberg, R. McDuff, C. P. Smith, H. RubinszteinDunlop, and M. J. Wegener, Opt. Quantum. Electron 24, S951 (1992).

[22] M. W. Beijersbergen, L. Allen, H. Van der Veen, and J. Woerdman, Opt. Commun. 96, 123 (1993).

[23] M. Beijersbergen, R. Coerwinkel, M. Kristensen, and J. Woerdman, Opt. Commun. 112, 321 (1994).

[24] L. Marrucci, C. Manzo, and D. Paparo, Phys. Rev. Lett. 96, 163905 (2006).

[25] Y. Shi, B. Shen, L. Zhang, X. Zhang, W. Wang, and Z. Xu, Phys. Rev. Lett. 112, 235001 (2014).

[26] A. Leblanc, A. Denoeud, L. Chopineau, G. Mennerat, P. Martin, and F. Quéré, Nat. Phys. 13, 440 (2017).

[27] J. Vieira, R. Trines, E. Alves, R. Fonseca, J. Mendonça, R. Bingham, P. Norreys, and L. Silva, Nat. Commun. 7, 10371 (2016).

[28] T. D. Arber, K. Bennett, C. S. Brady, A. Lawrence-Douglas, M. G. Ramsay, N. J. Sircombe, P. Gillies, R. G. Evans, H. Schmitz, A. R. Bell, and C. P. Ridgers, Plasma Phys. Controlled Fusion 57, 113001 (2015).

[29] G.-Z. Sun, E. Ott, Y. Lee, and P. Guzdar, Phys. Fluids 30, 526 (1987).

[30] D. Bauer, P. Mulser, and W. H. Steeb, Phys. Rev. Lett. 75, 4622 (1995).

[31] L. Rego, J. S. Román, A. Picón, L. Plaja, and C. HernándezGarcía, Phys. Rev. Lett. 117, 163202 (2016).

[32] H. Sun, Z. Xu, and C. Gao, Adv. Mater. 25, 2554 (2013).

[33] Y. Jiang, Z. Xu, T. Huang, Y. Liu, F. Guo, J. Xi, W. Gao, and C. Gao, Adv. Funct. Mater. 28, 1707024 (2018). 